\title{
Une mentalité du XIle siècle : Galbert de Bruges
} Jean Dhondt

\section{Citer ce document / Cite this document :}

Dhondt Jean. Une mentalité du XIle siècle : Galbert de Bruges. In: Revue du Nord, tome 39, n¹54, Avril-juin 1957. pp. 101109;

doi : https://doi.org/10.3406/rnord.1957.2233

https://www.persee.fr/doc/rnord_0035-2624_1957_num_39_154_2233

Fichier pdf généré le 07/04/2018 


\title{
UNE MENTALITÉ DU DOUZIEME SIECLE. GALBERT DE BRUGES
}

\author{
A Monsieur Félix ROUSSEAU, \\ en hommage a l'occasion de son éméritat.
}

Dans la mesure où l'essentiel de la civilisation dite occidentale est en cours de diffusion dans le monde entier, et, en liaison avec les civilisations régionales, influence profondément le mode de vie de toutes les populations de la planète, il est intéressant de se pencher sur ses origines. Il est bien certain que l'Europe occidentale ne fut pas de tout temps prédestinée à engendrer cette combinaison originale d'éléments divers qui lui assura progressivement la prédominance, sinon politique et matérielle (ce qui fut assez fugitif), mais civilisatrice, à travers le monde entier; il ne peut pas davantage être tenu pour assuré que cet état de chose soit très durable. Mais s'il est convenu que tout passe, il est tout à fait certain, aussi, que la civilisation dite occidentale fut la première à s'être répandue sur toute la planète. Dès lors, il est légitime de se demander à quel moment son originalité commença à s'affirmer. Sur ce point, on est fort desservi par la périodisation classique de l'histoire en Antiquité, Moyen Age, Epoque moderne et contemporaine. Cette subdivision efface, en effet, tolite trace de l'époque qui, à mon sens, marque le tournant peut-être décisif, ou l'un des deux ou trois tournants décisifs, dans l'évolution de notre civilisation. C'est au XII $I^{\mathrm{e}}$ siècle que $\mathrm{j}$ 'entends faire allusion. Étant bien entendu qu'une indication de date, même quand elle s'exprime par un siècle, est toujours relative là où il s'agit de phénomènes humains généraux, il me paraît bien que le XII ${ }^{\mathrm{e}}$ siècle donne vraiment le départ du monde moderne : à cettc ćpoque le monde caractérisé par la prépondérance accentuée des économies agraires voit s'affirmer avec force les populations urbaines, phénomène qui se développa au point que, de nos jours, le monde est réparti en zones à prédominance agraire - ce sont les pays "sous-développés" -, et zones de civilisation urbaine - ce sont les régions qui dominent économiquement et politiquement le monde. C'est l'époque, au surplus, où les structures féodales, génératrices de morcellement, commencent à céder la place à l'organisation bureaucratique. donc à la centralisation, donc à la possibilité de constituer de grands Etats unifiés; or, on sait que les principes nouveaux d'organisation (importance de l'écrit, spécialisation des fonctionnaires, bureaucratie), qui préfigurent le " manageriat" moderne, renversèrent la tendance à l'éparpillement en direction de la concentration. Et faut-il dire que la concentration, dans le domaine politique comme dans le domaine économique, est la caractéristique de notre civilisation ?

Enfin, pour s'en tenir à trois aspects seulement, il semble qu'au $X I I^{e}$ siècle s'affirme avec force le recul des tendances théocratiques et de la théologie comme science prédominante. Plus précisément, les hommes commencent à voir (lentement et prudemment) le Monde comme régi par des lois plutôt que par une volonté arbitraire.

Que l'on m'entende bien : ces différents aspects ne sont point nouveaux au XIIe siècle : on les rencontre à différentes époques antérieures, et dans d'autres formes de civilisation que la nôtre. Ce que je veux dire, c'est qu'à partir du XII ${ }^{e}$ siècle, il se fit un développement continu, selon ces lignes, à l'intérieur du monde occidental, c'est-à-dire un développement progressif vers la civilisation actuelle. Développement progressif, 
faut-il le dire, ne veut pas dire développement constant, sans ralentissements ni recul. Les sociétés humaines ne se développent jamais selon une ligne droite. Du moins, dans notre société, n'y eut-il plus jamais rupture définitive avec les grandes tendances auxquelles $\mathrm{j}$ 'ai fait allusion plus haut : depuis le XII ${ }^{\mathrm{e}}$ siècle l'évolution se dessine, à des rythmes changeants, mais sans hésitation fondamentale, vers les formes modernes de techniques, d'organisation et de pensée.

Or, nous sommes assez fortunés de posséder ce qu'on pourrait presque appeler le journal intime d'un homme qui vécut aux origines mêmes de cette évolution. Il s'agit du "Récit du meurtre de Charles le Bon " par Galbert de Bruges, écrit en 1127-28 1 .

L'ouvrage constitue l'un des classiques de l'histoire du Moyen Age, et il a été utilisé par un très grand nombre d'historiens, non seulement pour l'étude de problèmes aussi classiques que l'histoire de la féodalité ou l'histoire urbaine, mais aussi pour l'histoire de l'architecture, celle de l'art militaire et de la poliorcétique. J'ignore si d'autres auteurs ont déjà entrepris d'analyser Galbert en vue de l'étude des origines du rationalisme. Je n'en connais pas, et c'esì pourquoi je me propose de le faire ici.

Cependant, il faut bien s'entendre : à certains égards, il apparaîtrait presque ridicule de voir en Galbert un précurseur du rationalisme : comme je le montrerai en premier lieu, Galbert est superstitieux, et à cet égard il y a des choses curieuses à extraire de son récit. Pourtant, je ne crois pas trop téméraire de dire que le récit de Galbert montre précisément les premiers balbutiements de la pensée rationnelle en lutte avec les traditions. Un homme dont l'esprit est naturellement porté au raisonnement logique s'efforce de concilier les exigences de son cerveau avec les influences déterminantes de son milieu profondément religieux. Et, c'est ce qui rend le cas, à mon sens, particulièrement attachant, il ne s'agit point ici d'un penseur professionnel, d'un philosophe ni d'un théologien, mais de celui qu'à l'échelle de son époque nous pourrions appeler un "intellectuel moyen". C'est cela qui donne à son témoignage, me semble$t$-il, une exceptionnelle valeur.

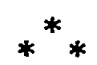

On ne sait pratiquement rien d'autre sur Galbert ${ }^{2}$ que ce que l'on peut dégager de son récit. De ce dernier, il résulte qu'il était Brugeois, ou du moins totalement identifié au milieu brugeois, et qu'il était probablement ecclésiastique. De son métier, il était " notaire ", c'est-à dire fonctionnaire de la chancellerie ou de l'administration domaniale du comte de Flandre. Il faut se rappeler que la chancellerie (c'est-à-dire le service où on rédigeait les chartes comtales), l'administıation des domaines du comte de Flandre, et, enfin, le chapitre de Saint-Donatien à Bruges constituaient un tout, dont Galbert faisait donc partie.

Dans une large mesure, l'intérêt extraordinaire du " Récit du meurtre de Charles le Bon " réside dans le fait qu'il s'agit d'un récit rédigé au jour le jour, et qui ne fut pas ensuite remanié en un tout cohérent. Galbert raconte les faits tels qu'il les a connus le jour même, et non pas tels qu'ils se présentent, finalement, à l'esprit d'un homme qui serait en possession de tous les éléments. Non seulement il raconte les faits, mais il prend position à leur égard sous l'impulsion du moment. Cela se traduit de la

1. L'édition la plus récente est celle de H. Pirenne, Histoire du Meurtre de Charles le Bon (Paris, Collection de textes pour servir a l'enseignement de l'histoire dite "Collection Picard" 1891).

2. Voir l'Introduction faite par Pirenne, pour l'édition susdite. 
manière la plus frappante dans les multiples contradictions de ses attitudes : pour donner un exemple banal, Galbert " change de parti " à tout moment dans la lutte qui se déroule en Flandre. Ce phénomène a été relevé par tous ceux qui se sont intéressés à son récit. Mais, comme j'espère le montrer, Galbert ne change pas seulement de parti à l'égard des différents prétendants au trône de Flandre; il modifie aussi son attitude en ce qui concerne l'interprétation fondamentale des événements, où réside précisément le problème de l'intervention de Dieu dans la destinée des hommes. C'est par là qu'il apporte un témoignage exceptionnel sur la mentalité des hommes de son temps.

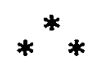

Rappelons brièvement ce qui s'est passé en Flandre en 1127. Le comte de Flandre Charles le Bon, petit-fils du comte Robert le Frison, est assassiné le 2 mars 1127 par des membres d'une famille de chevaliers, les Erembauld. Comme il ne laisse pas d'enfants, une lutte de succession s'engage parallèlement à la lutte menée par les barons de Flandre contre les meurtriers. Dans le grand nombre des prétendants au trône trois importent à notre exposé : Guillaume Cliton, fils du duc de Normandie Robert Courteheuse et qui appartient à la dynastie comtale flamande par sa grand-mère, sœur de Rohert le Frison; Baudouin, comte de Hainaut, issu de Baudouin VI de Flandre, frère aîné de Robert le Frison (donc de la branche aînée dépossédée par Robert le Frison); enfin Thierry d'Alsace, qui descend d'une fille de Robert le Frison ${ }^{1}$.

Guillaume Cliton l'emporte d'abord : il est élu comte de Flandre. Peu de temps après, les meurtriers, qui s'étaient réfugiés dans la tour de l'église Saint-Donatien de Bruges, sont faits prisonniers et précipités, vivants, du haut de cette tour. Quelques mois plus tard, les villes de Flandre, mécontentes du gouvernement de Guillaume Cliton, se soulèvent contre lui et reconnaissent pour comte Thierry d'Alsace. Une lutte longue et indécise s'engage entre les deux rivaux; succès et revers se balancent d'abord, mais peu à peu Guillaume Cliton prend l'avantage. Il est toutefois tué lors du siège d'Alost, ce qui met fin à la lutte au bénéfice de Thierry d'Alsace.

Galbert.

$\mathrm{Ce}$ sont ces événements qui constituent la substance du récit de

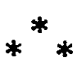

Nous tenterons maintenant, - c'est l'objet de la présente étude de cerner d'aussi près que possible la mentalité du narrateur. A cette fín.

1. Voici le tableau généalogique des comtes de Flandre :

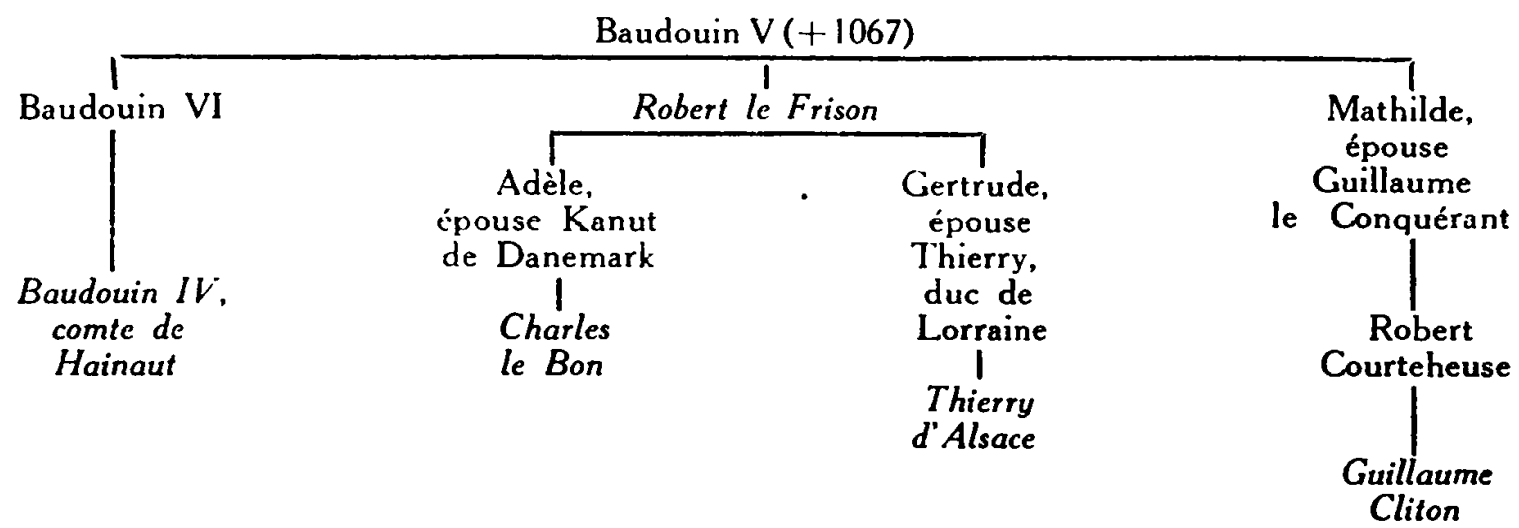


nous pouvons reconnaître successivement deux personnages opposés, et qui pourtant coexistent certainement en lui : le Galbert superstitieux et le Galbert rationaliste.

Essayons d'abord, brièvement, de résoudre cette contradiction appa rente : Galbert appartient à une époque profondément superstitieuse, au sens le plus banal du terme, et il partage les superstitions de son époque. D'autre part, Galbert est un esprit étonnamment concret et positif ; avant tout, il veut " comprendre". Telle est la clef, extrêmement banale, de l'apparente contradiction de son esprit.

Nous indiquerons dans un instant, jusqu'où va la superstition de Galbert dans le domaine de la magie. Nous voulons nous placer, en premier lieu, sur un plan plus commun : Galbert, comme ses contemporains, croit dur comme fer aux présages, à toutes les formes de présages : les phénomènes astronomiques ${ }^{1}$, les rêves ${ }^{2}$, enfin les "accidents " ${ }^{3}$. Pour ce qui est de ce dernier cas, on est amené à penser que les architectes de ce temps-là étaient de bien mauvais bâtisseurs, et qu'ils avaient bien de la chance que l'époque crût aux présages : on demeure, en effet, un peu épouvanté de la facilité avec laquelle les constructions s'écroulaient.

Donc, Galbert est superstitieux. Mais, comparé à ses contemporains, il semble l'avoir été plutôt moins qu'eux. C'est ce qu'on peut inférer de son attitude devant le miracle. Galbert, bien entendu, croit au miracle. Nous ne voudrions pas formuler le moindre doute à cet égard : Galbert est très religieux. Mais son esprit positif reconnaît moins facilement un miracle dans telle situation extraordinaire que ne le font ses contemporains. Cela peut être prouvé facilement, parce que nous possédons, à côté du récit de Galbert, plusieurs autres récits du meurtre de Charles le Bon : celui de Gauthier de Thérouanne, celui de Suger, celui de Herman de Tournai

Donnée intéressante sur la mentalité de l'époque : il est visible que le récit du meurtre s'est instantanément compliqué, dans l'imaoination populaire, de plusieurs faits réputés miraculeux. Il faut noter que le comte avait été assassiné pendant qu'il priait devant l'autel, ce pourquoi il fut réputé martyr et devint bientôt l'objet d'un culte. Quoi qu'il en soit, nous constatons que les différents récits consacrés à ces événements mentionnent tous divers miracles. Il en est de même chez Galbert, mais seulement dans un seul cas : la guérison d'un paralytique de naissance qui s'était appliqué le sang du comte-martyr ${ }^{4}$. Mais, et c'est ceci qui est important, si les autres "miracles" rapportés par les différents auteurs sont également rapportés par Galbert, il ne les présente point comme des miracles ${ }^{5}$. Il est donc bien évident qu'il est beaucoup plus critique que les autres auteurs dans le domaine du miraculeux. C'est là une indication précieuse sur sa mentalité, mais qui, en somme, n’apparaît pas très surprenante à quiconque est familiarisé avec cette mentalité. Je l'ai déjà dit, je le répète : Galbert est un esprit extraordinairement concret et positif, on pourrait presque dire pédagogique. C'est ce qui a rendu son récit

1. Ibid., ch. 2 , p. 5 (une éclipse).

2. Ibid., ch. 84, p. 129.

3. Ibid., id. et ch. 102, p. 148.

4. Ibid., ch. 22 , p. 39 . Le même événement chez Gauthier de Thérouanne (MGH., SS.. XII), ch. 30, chez Herman de Tournai (MGH, SS., XIV), ch. 29.

5. Le fait que les aliments n'avaient plus aucun goût pour les assiégés, chez Galbert, ch. 73 ; chez Gnuthier, ch. 46 ; chez Suger, Vie de Louis le Gros, ch. 130 (éd. Waquet, p. 246).

Le fait que le cadavre de Charles le Bon ne répandait aucune odeur, chez GALBERT, ch. 77 : chez Gauthier, ch. 47 ; Herman, ch. 35. 
tellement précieux pour les différentes branches spéciales de l'histoire : rapporte-t-il une prestation d'hommage, il expose successivement tous les gestes, fournissant ainsi l'un des exposés les plus précis que l'on possède de cette cérémonie ${ }^{1}$; narre-t-il une bataille entre armées de chevaliers, il ne manque pas de décrire la disposition des armées et les manœuvres successives $^{2}$; de même s'il s'agit d'un duel ${ }^{3}$. Décrit-il un siège, il explique la construction des échelles. Bref, il est clair que Galbert veut comprendre et faire comprendre. Cela étant, il est impossible qu'il s'en tienne exclusivement à la succession explicative des faits matériels : la même attitude d'esprit va se manifester dans le domaine des faits extra-matériels.

Esprit donc à la fois superstitieux et positif, Galbert fait même preuve, une fois, d'une tournure d'esprit presque voltairienne. Il met une malice évidente à raconter l'histoire du prêtre si naif qui priait ardemment et dévotement devant des vases précieux qu'on lui avait dit remplis de reliques insignes, mais qui étaient parfaitement vides et constituaient le produit d'un vol 4 .

Venons en maintenant à la magie, un des aspects les plus étonnants de ce récit, un des plus instructifs aussi pour notre connaissance de la mentalité de l'époque. Comme on l'a vu plus haut, il se produisit à un moment donné la situation suivante : les villes s'étant soulevées contre le nouveau comte, Guillaume Cliton, reconnurent pour comte Thierry d'Alsace. La lutte s'équilibra et s'éternisa. Il est remarquable qu'à ce moment précis, des sorciers entrent en scène. Galbert signale d'abord, par ouï dire évidemment, qu'à Gand et à Lille des enchanteurs commencèrent à jeter des sorts sur les compétiteurs au trône comtal ${ }^{5}$. Mais bientôt il ne s'agit plus d'ouí-dire : nous arrivons au 12 juin 1128 , journée dans laquelle le parti de Thierry d'Alsace (celui de Bruges et de Galbert) remporta divers succès. Ce n'est pas cela qui importe ici, mais bien la manière dont Galbert rapporte, ou plutôt explique, ces succès ${ }^{6}$. "Les habitants de Bruges, écrit-il, attribuèrent ces succès à un de leurs prêtres, qui avait jeté l'anathème sur Guillaume Cliton et son parti. A Ypres (ville tenant le parti de Guillaume Cliton, l'ennemi des Brugeois), il y avait un certain prévôt, Hildfredus, qui jetait chaque jour l'anathème sur le parti de Thierry, notre comte. Mais cette fois-ci, l'anathème de notre prêtre était le plus fort, et je ne pense pas que notre prêtre cessera ses anathèmes avant que le comte Guillaume et les siens et son prévôt Hildfredus soient partis pour l'exil. Et n'est-il pas prodigieux qu'un prêtre puisse ensorceler (incantare) Dieu au point que, Dieu le voulut-il ou non, il chasse Guillaume du comté".

La dernière phrase n'est-elle pas prodigieuse ? Quelles perspectives n'ouvre-t-elle pas sur les croyances profondes de ces hommes ? Le sorcier peut donc lier Dieu par ses sortilèges?

Que telles soient bien les convictions prévalentes à l'époque est confirmé tant par la suite des événements que par les réactions de Galbert. Dans les jours suivants, la guerre entre les deux prétendants au trône va devenir de plus en plus une lutte entre les magiciens des deux partis. Cette magie est, on le voit, étroitement liée aux pratiques religieuses : ce sont des prêtres qui l'exercent, dans le passage cité ci-dessus et dans

1. Galbert, ch. 56 , p. 89.

2. Ch. 114, pp. 163-164.

3. Ch. 58 , p. 94.

4. Ch. 61 , p. 100.

5. Ch. 110, p. 157 (Lille), ch. 112, p. 139 (Gand).

6. Ch. 113, p. 161 . 
d'autres subséquents, et, par surcroît, les pratiques magiques sont étroitement liées à des austérités outrées, qui, on va le voir, indignent curieusement Galbert.

Les succès des Brugeois sont passagers : peu de jours après, exactement le 21 juin, Guillaume Cliton remporte sur Thierry d'Alsace un important succès à Axpoele ${ }^{1}$. Avant la bataille, Guillaume et ses chevaliers firent pénitence, se coupèrent les cheveux et se dépouillèrent de leurs vêtements, ne'conservant que leur chemise et leur cuirasse. Quand les Brugeois, vaincus dans la bataille, eurent connaissance de ces détails, ils s'empressèrent de se couper les cheveux eux aussi et de se dépouiller de leurs vêtements. Les prêtres brugeois prêchèrent la pénitence, firent une procession et jetèrent solennellement l'anathème sur le parti de Guillaume Cliton.

A ce propos, Galbert fait la remarque suivante" : "Il faut noter que nos excommunications et celles de l'archevêque (de Reims, qui avait excommunié le parti de Thierry d'Alsace) luttaient les unes contre les autres". Il y a là, je crois, plus qu'une simple constatation. Il y a un commencement de doute qui s'installe en son esprit. En effet, il va bientôt désapprouver formellement toutes ces pratiques, et de la manière la plus énergique $^{3}$ : "Nos prêtres affirmaient stupidement (idiote) que c'étaient les sortilèges (incantationes) du curé d'Aartrijke et du curé de Koekelare et du clerc Odfrid qui avaient mis en fuite le comte Thierry, alors que c'est Dieu qui dispose et règle tout". Ce dernier membre de phrase constitue, on le voit, exactement le contrepied du point de vue que Galbert avait défendu plus haut, lorsqu'il admettait qu'un enchanteur était capable de forcer Dieu à lui obéir. II est clair que Galbert est partagé entre sa croyance à la sorcellerie d'une part, en l'omnipotence divine de l'autre. Dans le premier cas, il cède, c'est visible, aux croyances prédominantes en son milieu. Dans le second cas, où il s'est repris, j'oserais dire qu'il assume un point de vue plus rationaliste ; en tout état de cause, les dogmes d'une religion fortement établie et systématisée sont plus acceptables pour un esprit rationnel que la croyance aux enchantements.

Il est peut-être opportun de faire remarquer que dans l'intervalle entre ses deux prises de position opposées, Galbert, a changé de parti dans la lutte entre les deux prétendants, il était partisan de Thierry d'Alsace à l'époque où il affirmait sa confiance dans les enchanteurs, il est devenu un partisan de Guillaume Cliton au moment où il refuse de croire à la force des sortilèges ${ }^{4}$. Y a-t-il un lien entre les deux séries de faits ? Il me semble que oui : Galbert ne veut-il pas se persuader que les sortilèges sont sans force, précisérnent parce qu'il craint que les Brugeois, à force de sortilèges, ne fassent encore changer la victoire de camp ? Car en effet, à ce moment, les Brugeois éperdus ne savent plus qu'inventer dans le domaine du surnaturel pour obtenir la victoire, et Galbert estime que ces croix portées en procession "magis Deum ad iram quam ad placationem provocaverunt ${ }^{5}$ ", et il s'en prend maintenant ${ }^{6}$ avec violence à ces "multi divinatores et laici et sacerdotes qui adulabantur civibus nostris, predicentes eis quaecumque sciebant cives voluisse audire", qui imposent des jeûnes extraordinaires, jettent à nouveau l'anathème sur le comte Guillaume... et s'en-

1. Ch. 114, pp. 162-64.

2. Id., p. 165.

3. Ch. 115, p. 166.

4. Du moins il l'est certainement devenu au chapitre suivant, ch. 116.

5. Ch. 116, p. 167.

6. Ch. 118, pp. 169-70. 
richissent ainsi.." N'est-ce pas encore là un passage révélateur, jusque dans son explosion anticléricale? N'est-il pas profondément révélateur du trouble de Galbert lui-même, déchiré dans ses convictions les plus profondes et jeté par son attitude individualiste hors de la communauté humaine à laquelle il s'est si profondément intégré, mais dont il ne partage plus l'allégeance politique et dont il se sépare dans le domaine des croyances aux moyens surnaturels destinés à assurer la victoire, cependant qưil ne peut se soustraire aux conséquences des attitudes de révolte tant dans le domaine séculier que dans le domaine ecclésiastique (les Brugeois refusent de se soumettre à l'interdit que l'archevêque a jeté sur la ville) ${ }^{1}{ }^{1}$. C'est un déchirement total et profond.

Cet exposé sur l'emploi de la magie dans la lutte entre les deux rivaux à la couronne de Flandre nous a révélé, d'une part, combien forte était la croyance à la magie, à peine voilée sous un masque religieux. Elle nous a montré aussi Galbert partagé entre la croyance commune et une conception plus élevée des forces qui agissent sur les hommes, et à laquelle il se rallie finalement, au prix d'un douloureux arrachement aux croyances de son milieu social. Déjà il y a là l'affirmation d'un individualisme certain et d'un rationalisme encore peu perceptible dans le domaine religieux. Nous allons voir maintenant ce rationalisme s'affirmer avec beaucoup plus de force, mais aboutissant encore une fois à une crise de conscience.

Rappelons d'abord, plus expressément que nous ne l'avons fait jusqu'à présent, que, pour Galbert, Dieu régit expressément tous les actes des hommes. C'est là une conception sous-jacente à tout son récit et qui s'exprime presqu'à chaque page. Cette conception, faut-il le dire, n'offre rien d'extraordinaire. Où la difficulté commence, c'est lorsque Galbert prête à Dieu sa propre logique et veut, par conséquent, retrouver dans les événements des séquences intelligibles à son propre esprit.

Il est assez remarquable que Galbert vise à intégrer l'ensemble des événements dans un plan unique, plan divin, naturellement, et qui se ramène au châtiment divin inéluctable de ceux qui ont commis le mal.

Voici ce plan : en 1071, Robert le Frison a commis un crime inexpiable en s'emparant du trône de Flandre au détriment de ses jeunes neveux après avoir, sur les saintes reliques, juré, dans l'église de SaintDonatien même, de ne jamais rien entreprendre contre eux.

De son côté, la puissance des Érembauld résulte, elle aussi, d'un crime abominable : le fondateur de la dynastie, Erembauld, amant de la femme du châtelain de Bruges son suzerain, a assassiné son maître en le "précipitant" (le point a son importance) dans la mer.

Par surcroît, les Erembauld ont aidé Robert le Frison à commettre son crime. Il y a donc deux lignées criminelles, par surcroît alliées ${ }^{2}$.

Les événements de 1127 sont, pour Galbert, la vengeance divine punissant simultanément les deux forfaits sur les descendants des deux

I. Voir les lamentations de Galbert, ch. 116, p. 167 (Notandum est...).

2. Lexposé de toute cette conception se trouve aux ch. 69 a 71 . On se demande combien il y a en tout cela raisonnement propre au seul Galbert et légendes populaires en circulation. Le récit de l'assassinat de Boldran par Erembauld ne peut guère avoir d'autre origine qu'une légende populaire, puisque le meurtre se produisit, au dire même de Galbert, strictement sans témoins. Que Galbert présente la prise du pouvoir par Robert le Frison comme une "trahison " est également très curieux. Il est assez douteux que cette conception lui soit personnelle; d'ailleurs. dans son récit on rencontre des éléments, notamment l'histoire de l'émissaire qui se faisait passer pour aveugle, qui ont bien un parfum de légende populaire. Au-dessous, si l'on peut dire, des exposés officiels sur l'histoire des comtes de Flandre, tels que nous les avons gardés par exemple dans la Genealogia Bertiniana, il circulait donc dans le peuple des récits moins fatteurs sur la dynastie. 
criminels : le comte Charles le Bon, descendant de Robert le Frison, a été assassiné, et cela dans l'église même, Saint-Donatien, où Robert le Frison s'était parjuré. Il a été assassiné par les descendants d'Érembauld, et en conséquence, ceux-ci ont été précipités à leur tour (ils ont été mis à mort après leur capture, en étant jetés vivants du haut d'une tour).

Tout cela constitue donc un ensemble cohérent, qui a dû satisfaire l'esprit logique de Galbert.

Cet esprit logique a trouvé, d'ailleurs, d'autres motifs encore de satisfaction. Plusieurs des principaux "barons" de Flandre avaient été plus ou moins complices des meurtriers; ils étaient toutefois trop puissants pour qu'on les punisse. Or, voilà qu'ils meurent l'un après l'autrc, dans l'année même, à la suite d'accidents banals. C'est bien la vengeance divine qui continue à se développer. Galbert exulte ! ${ }^{1}$.

Voilà qui est parfait. On pourrait peut-être objecter qu'un comte aussi admirable, selon Galbert, que Charles le Bon ne méritait pas d'être l'objet du châtiment divin; mais le narrateur s'est très probablement rassuré par la pensée - souvent répétée dans son récit - qu'en mourant martyr de la justice, le comte Charles accédait d'emblée à la béatitude éternelle.

Il subsistait encore un petit élément de trouble dans les perspectives de Galbert : ne serait-il pas logique qu'après la mort de Charles le Bon, le trône de Flandre revienne aux descendants de la branche spoliée en 1071, c'est-à-dire au comte de Hainaut Baudouin IV ? Il ne fait point de doute que Galbert ait pensé ainsi, car à plusieurs reprises, à l'époque où la lutte pour la succession de Charles le Bon s'engageait, il ne manque point de qualifier le comte de Hainaut de justius heres, de successeur légitime ${ }^{2}$.

Or, ce ne fut point Baudouin de Hainaut qui accéda au trône de Flandre, mais bien Guillaume Cliton. Galbert a pu se rassurer assez aisément : en effet, Guillaume Cliton ne descendait point de Robert le Frison, mais d'une ligne plus ancienne de la dynastie.

Les événements se déroulaient donc bien, en gros, selon le plan logique prêté par Galbert à Dieu, jusqu'au 6 avril 1128. Que s'est-il donc passé ? Voici : Lambert de Reddenbourg, un membre de la dynastie des Erembauld, c'est-à-dire des meurtriers du comte Charles, était soupçonné de culpabilité. Or, il établit son innocence par l'épreuve du fer chaud ${ }^{3}$. Comme Galbert le tenait pour coupable, le résultat de l'épreuve a dû le troubler, mais il n'en apparaît rien dans son texte. Ce n'était toutefois que partie remise. Vöilà en effet que le 30 avril, c'est-à-dire moins d'un mois après l'épreuve du fer chaud (6 avril) Lambert est tué ${ }^{4}$. Cette fois, c'est l'affolement chez Galbert, car enfin, ou bien Lambert est coupable, et alors, comment expliquer l'issue de l'épreuve du fer chaud, ou bien il est innocent, et alors pourquoi Dieu permet-il qu'il soit tué quelques jours après?

Notre auteur cherche frénétiquement une explication ${ }^{5}$. Il finit par la trouver, mais on à peine à croire qu'elle ait pu le satisfaire. Lambert, dit-il, était bien certainement coupable du meurtre de Charles le Bon, mais il s'est repenti de ce crime et pour ce motif Dieu lui a permis de triompher

1. Ch. 89 et 91 .

2. Ch. 67 : comes de Montibus... regnum Flandriarum, quod sibi jure cognationis justius pertinebat. Cf. aussi, ch. 19.

3. Ch. 105.

4. Ch. 108.

5. Ibidem. 
de l'épreuve du fer chaud. Ensuite, il a commis une nouvelle faute grave : ayant juré d'observer la paix, il est allé combattre à nouveau ses ennemis particuliers. Dieu l'a donc puni.

Ce raisonnement implique, faut-il le dire, que l'épreuve du fer chaud n'établit point linnocence de celui qui s'y soumet avec succès. Le lecteur se rend bien compte des infinies complications que cela entraîne sur le terrain de la procédure en matière criminelle. Galbert s'en est rendu compte lui aussi, et il souligne expressément la différence qui existe, donc, entre des épreuves du type de celle du fer chaud et le duel judiciaire: dans ce dernier, fait-il remarquer, l'innocent est vainqueur et le coupable vaincu, il n'y a point d'autre issue possible et donc cette procédure donne toute garantie. Dans l'épreuve du fer chaud, au contraire, le coupable, s'il se repent, sera épargné.

L'intérêt de ce passage est qu'il montre Galbert amené par la logique de ses raisonnements à prendre position dans le domaine, très particulier, de la procédure criminelle, et cela, notons-le, au moment où, en effet, le problème commençait à se poser en Flandre, plus particulièrement dans les villes.

Quoi qu'il en soit, il demeure que, pour la première fois, les conceptions de Galbert au sujet du plan divin sont soumises à une rude épreuve. Mais ce n'est qu'un début : le 27 juillet, Cuillaume Cliton est tué au siège d'Alost. et dès lors Thierry d'Alsace a partie gagnée. Cette fois, c'est l'effondrement.

En effet, la victoire de Thierry, c'est, à tous égards, l'anéantissement des conceptions de Galbert : celui-ci ne peut pas en effet se faire d'illusions : la victoire de Thierry, c'est bien aussi la victoire des Erembauld. Tous ceux qui ont survécu à la répression se sont hâtés, en effet, de rejoindre l'ennemi de Guillaume Cliton, et, Galbert le constate avec amertume, sans qu'on ait osé les citcr en justice". Donc, les descendants du " traître" Erembauld triomphent. Mais ce n'est pas tout : les descendants du "traître" Robert le Frison triomphent aussi, puisque ce n'est ni le comte Baudouin IV de Hainaut, descendant de la branche dépossédée, qui accède au trône de Flandre, ni Guillaume Cliton, descendant d'une branche plus ancienne; c'est bien, entre tous les candidats, celui-là précisément qui descend de Robert le Frison, Thierry d'Alsace, qui est reconnu pour comte de Flandre!

Combien tout cela a troublé Galbert, les derniers chapitres ${ }^{2}$ de son œuvre le prouvent. Voici en effet jusqu'où son sens de la logique combiné avec sa croyance profonde en la logique divine pousse le raisonnement: cum per mortem alterius Deus pacem vellet restituere patriae, cur magis dispensavit, ut moreretur Willemus comes, qui justiorem causam regendi terram obtinuit, et quare non citius mortuus fuit Theodericus, qui injuste superpositus videbatur, aut qua justitia Deux concessit ei consulatum, qui violenter arripuit dignitatem. Si igitur neuter eorum bene suscepit comitatum, jure uterque erat auferendum.

A ces questions, Galbert répond par un raisonnement embrouillé et fallacieux, qui n'a pu le satisfaire que parce que son esprit, à la fois logique et religieux, exigeait absolument une réponse conciliant les deux aspects. Son ouvre se termine là, mais elle a été poussée assez. loin pour que nous ayons pu saisir comment un homme moyen du XII e siècle luttait pour concilier la foi et la raison.

Gand, Faculté de Philosophie et Lettres.

Jean Dhondt.

1. Ch. 110 , p. 157.

2. Ch. 120, et surtout 121 . 